Check for updates

Cite this: RSC Adv., 2017, 7, 35154

Received 30th May 2017

Accepted 7th July 2017

DOI: $10.1039 / \mathrm{c} 7 \mathrm{ra06036j}$

rsc.li/rsc-advances

\title{
Improving photocatalytic oxygenation mediated by polymer supported photosensitizers using semiconductor quantum dots as 'light antennas' $\dagger$
}

\author{
Víctor Fabregat, M. Isabel Burguete, Santiago V. Luis (D) * and Francisco Galindo (D) * \\ Quantum dots (QDs) adsorbed on the surface of polymeric photocatalysts improved the photochemical \\ generation of singlet oxygen due to the efficient energy transfer process from the semiconductor \\ nanoparticles to the bound organic photosensitizer (rose bengal).
}

There is an urgent need to find sustainable methods to carry out chemical reactions in order to meet the increasing demands of industrialized societies. Solar light is the primary source of energy and hence methods and materials employing photocatalytic strategies are gaining popularity. ${ }^{1}$ But the full electromagnetic spectrum irradiated by the Sun is not properly harnessed by most photocatalytic materials. The paradigmatic example of this situation is titanium dioxide, a semiconductor material studied for more than 40 years and used in a long list of applications, which suffers from the important drawback of not absorbing visible light (400-800 $\mathrm{nm})$ and hence it must be activated using UVA light (315-400 nm). ${ }^{2}$ Taking into account this limiting issue, basically three strategies have been followed: (1) doping of $\mathrm{TiO}_{2}$ with elements yielding materials absorbing in the visible range; (2) use of new colored inorganic architectures not based on titania (iron or bismuth based materials, for instance); and (3) use of organic photocatalysts (photosensitizers) either soluble or supported on a variety of matrices. As recently pointed out by Lacombe and Pigot, ${ }^{3}$ the use of organic photosensitizers attached to a solid matrix has the main advantage of using visible light for the excitation but other advantages can also be envisaged as are the possible tuning of the absorption wavelength, as well as that of the hydrophilicity, porosity and adsorptive features of the support, and the elimination or significant reduction of the aggregation of the photosensitizer. In this regard, the list of selective photo-oxygenations mediated by supported photocatalysts is already extensive. ${ }^{1-3}$ However, there is still room for improvement. In many cases, even when using visible light part of the potentially useful radiation from the Sun is missing, since the corresponding photosensitizers absorb only in narrow wavelength ranges leaving important spectral windows with weak (or negligible) absorptions. For instance, porphyrins absorb strongly around $420 \mathrm{~nm}$ (Soret band), very weakly at $c a$. 550-600 nm (Q

Universitat Jaume I, Departamento de Quimica Inorgánica y Orgánica, Av. Sos Baynat, s/n, E-12071 Castellón, Spain.E-mail: luiss@uji.es; francisco.galindo@uji.es

$\dagger$ Electronic supplementary information (ESI) available: Experimental protocols, spectral features and representative irradiation. See DOI: 10.1039/c7ra06036j bands) and negligibly at $440-540 \mathrm{~nm}$ and above $650 \mathrm{~nm}$ (depending on the particular structure). A similar situation applies for all the organic photosensitizers, which display characteristic absorption bands but also well-defined valleys. ${ }^{4}$

Extending the range of excitation is a problem that was tackled in the field of photodynamic therapy of cancer (PDT). ${ }^{5}$ In PDT, laser light is used to generate cytotoxic reactive oxygen species (ROS), but sometimes it is not possible to use an excitation wavelength matching the maximum absorption of the photosensitizer. In a pioneering paper by the group of Burda, ${ }^{6}$ the absorption of a silicon phthalocyanine photosensitizer was extended with a CdSe semiconductor quantum dot. This finding represented a breakthrough later confirmed and developed by others. ${ }^{7}$ It is now widely accepted that the broad absorption of QDs can be used to sensitize (via Förster Resonance Energy Transfer) a neighboring photosensitizer, or in other words, to serve as 'light antennas' to capture energy of wavelength not usable directly by the photosensitizer. The field has evolved rapidly, leading to the publication of a vast number of papers analyzed in several reviews. ${ }^{8}$ Despite of the sensitization process above described, the advantages of this strategy seem to be applied almost only to the field of medical sciences (PDT and bioimaging) but not specifically to the use of light to promote catalytic oxidations.

In this communication we report the extension of the range of excitation of a certain polymer supported organic photosensitizer by using semiconductor quantum dots. By means of this procedure the production of singlet oxygen has been efficiently accomplished using a wavelength $(450 \mathrm{~nm})$ where the photosensitizer naturally does not absorb. To the best of our knowledge this is the first time that FRET sensitization via QDs is applied to a catalytic oxygenation mediated by a polymer supported photosensitizer. Contrary to the described ensembles used for PDT and bioimaging, where the QD is coated with the photosensitizer leading to a nanometric hybrid, in our case the QD is adsorbed on the surface of the micrometric polymeric photocatalyst. 
For this study, two photoactive polymers recently described by our group (Pc and Pa in Fig. 1) were used as photocatalysts. They are based on Rose Bengal (RB) covalently attached to a porous matrix of polystyrene and both have been shown to be efficient generators of singlet oxygen $\left({ }^{1} \mathrm{O}_{2}\right) \cdot{ }^{9}$ Numerous materials have been reported ${ }^{\mathbf{1 0}}$ able to generate photochemically ${ }^{1} \mathrm{O}_{2}$ for synthetic, ${ }^{1 \mathbf{1 1}}$ biological ${ }^{\mathbf{1 2}}$ or environmental applications. ${ }^{13}$ As sensitizing entities we used two types of semiconductor nanoparticles (quantum dots) made of CdSe with a protective shell of ZnS (core-shell CdSe/ZnS), which had also been used previously by us for a number of hybrid materials. ${ }^{14}$ The QDs differ in their size, or in other words, in the photoluminescence emission wavelength (480 $\mathrm{nm}$ for QD480 and $530 \mathrm{~nm}$ for QD530).

The rationale behind this approach was that an effective FRET process would take place leading to indirect excitation of the RB moiety when exciting the QD nanoparticle (Fig. 2), taking into account the overlapping between the emission of both QDS and the absorption of RB at the polystyrene matrix (see Fig. $\mathrm{S} 1 \dagger$ ). This energy transfer would lead to population of the triplet state of RB and concomitantly to the generation of singlet oxygen. The comparison of two polymers with different surface characteristics and two QDs with different optical properties would give a glimpse about the optimal combination to carry out photocatalytic oxidations.

Hybrids of QDs and Pc or Pa were prepared as indicated in the experimental section (see ESI $\dagger$ ). Hexadecylamine coated QDs are prone to precipitate in $\mathrm{MeOH}$ hence it was expected that in the presence of the microparticulated photocatalyst the deposition would occur at the surface of this material. The obtained mixtures had a colloidal appearance (Fig. 3). Microscopic analyses by Scanning Electron Microscopy (SEM) of the dried samples revealed that the morphology of the photocatalysts remained also
Pc

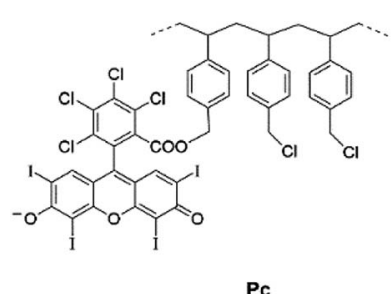

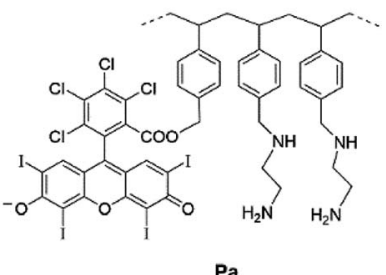

$\mathrm{Pa}$
Fig. 1 Structures of rose bengal grafted polymers used in this study.

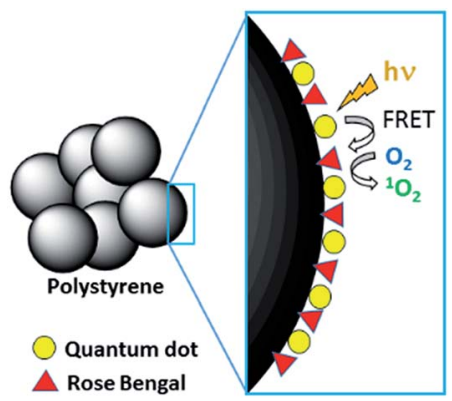

Fig. 2 Schematic representation of the double energy transfer process occurring at the surface of the photoactive polymers.
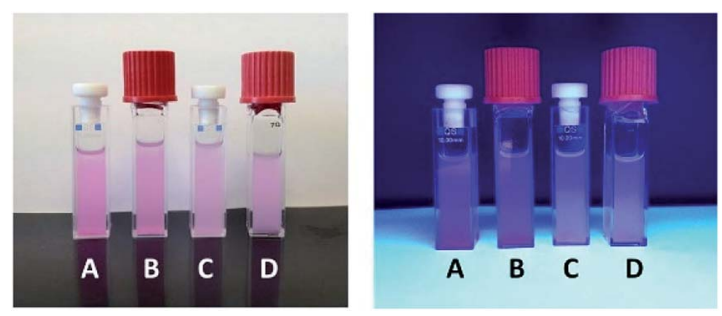

Fig. 3 Pictures of suspensions of QD480@Pc (A), QD530@Pc (B), QD480@Pa (C) and QD530@Pa (D) in methanol (4 $\left.\mathrm{mg} \mathrm{mL}^{-1}\right)$ under visible (left) and UV light (right).

unaltered as compared to naked Pa and Pc (Fig. 4). In order to confirm the full deposition of the nanoparticles at the surface of the polymers centrifugation of the samples was performed leading to supernatants with no trace of dissolved QDs.

The photocatalytic properties of the four hybrids QD480@Pc, QD530@Pc, QD480@Pa and QD530@Pa were tested using 9,10anthracenedipropionic acid (ADPA, $1.2 \times 10^{-4} \mathrm{M}$ ) in methanol, which is a well-known singlet oxygen trap, which transformation can be easily followed by UV-vis absorption spectroscopy (see Scheme S1 and Fig. S2 $\dagger$ ). ${ }^{9}$ The irradiations were conducted with a $450 \mathrm{~W}$ xenon lamp as the excitation source and a monochromator to select the $450 \mathrm{~nm}$ wavelength $( \pm 1 \mathrm{~nm})$ in order to excite selectively the adsorbed QDs.

Fig. 5 presents the conversions of ADPA after $5 \mathrm{~min}$ of irradiation of suspensions of the four mentioned hybrids at different proportions of QD relative to the polymer. This assay was performed starting from a fixed amount of Pc or Pa and adding increasing amounts of QDs in order to determine the optimal loading for the desired photoreaction. As it can be seen in Fig. 5, a loading of only $10.4 \mu \mathrm{g}$ of QD per mg of polymer caused measurable increases in the conversions of ADPA (in the case of Pc from $7-8 \%$ to $10-12 \%$ and in the case of Pa from 8-9 to 20-21\%). Upon incremental addition of QDs, in separate experiments, the conversions were steadily increasing, upon reaching $45-50 \%$ for Pa and 25$30 \%$ for Pc.
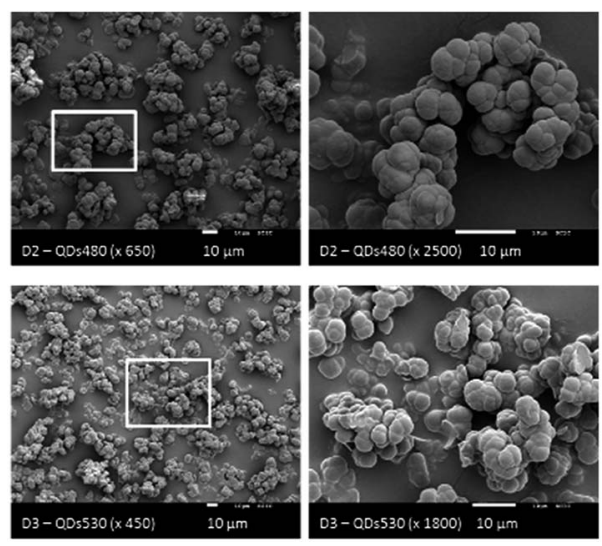

Fig. 4 Illustrative scanning electron microscopy (SEM) images of QD480@Pc (top, both images at different magnification) and QD530@Pa (bottom, both images at different magnification). 


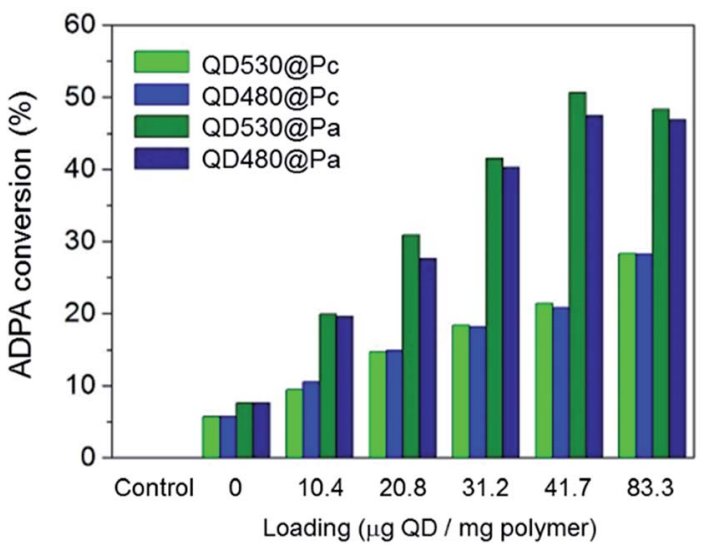

Fig. 5 Conversion of ADPA $\left(1.2 \times 10^{-4} \mathrm{M}\right.$ in methanol) after irradiation (5 min.) in the presence of hybrids QD480@Pc, QD530@Pc, QD480@Pa and QD530@Pa (4 $\mathrm{mg} \mathrm{mL}^{-1}$ ) with different load of QD Note that label 'control' corresponds to no polymer, and label ' $0 \mu \mathrm{g}$ $\mathrm{mg}^{-1}$ corresponds to non-modified polymers Pc and Pa. Excitation wavelength was $450 \mathrm{~nm}$.

Taking into account these data, a fixed value of $41.7 \mu \mathrm{g}$ per $\mathrm{mg}$ of polymer was selected as the loading of QDs in order to perform detailed kinetic determinations. Thus, the UV-vis absorption of ADPA was recorded after irradiation at $450 \mathrm{~nm}$ and fitted to a pseudo-first order kinetics, in agreement with the behavior of other 9,10-disubstituted anthracenes used as singlet oxygen traps. ${ }^{9}$ Fig. 6 displays the different kinetic traces obtained for the four hybrids along with the parent polymeric photocatalysts $\mathbf{P c}$ and $\mathbf{P a}$. The results using $\mathbf{R B}$ in solution have also been included for comparison. Note that all the irradiations were done at a wavelength where the absorption of RB is minimal. As a matter of fact, the slope for the homogeneous photosensitizer is almost negligible (red line in Fig. 6). In the same way, supported photosensitizers Pc and Pa performed the photooxidation of ADPA at a very slow rate. However, polymers doped with QDs carried out this

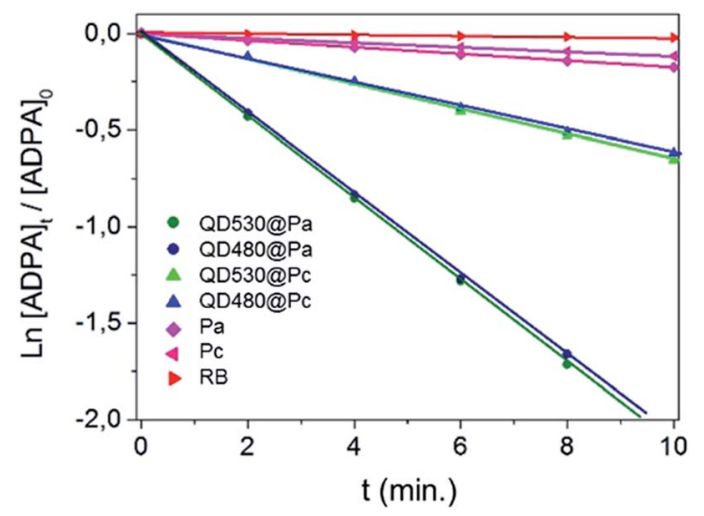

Fig. 6 Kinetic traces (pseudo-first order) for the oxygenation of ADPA in methanol photocatalyzed by QD480 @Pc, QD530 aPc, QD480@Pa and QD530@Pa (4 mg mL ${ }^{-1}$ ). Control reactions are also included (Pc, $\mathrm{Pa}$ and $5 \mu \mathrm{M}$ rose bengal). Fixed QD loading of $41.7 \mu \mathrm{g}$ QD per $\mathrm{mg}$ polymer. Excitation wavelength was $450 \mathrm{~nm}$.
Table 1 Summary of the photocatalyzed oxygenation of ADPA $(1.2 \times$ $10^{-4} \mathrm{M}$ ) in methanol under $450 \mathrm{~nm}$ irradiation

\begin{tabular}{llll}
\hline Support $^{a}$ & $\begin{array}{l}\text { Sensitizer }^{b} \\
\text { (donor) }\end{array}$ & $\begin{array}{l}\text { Photocatalyst } \\
\text { (acceptor) }\end{array}$ & $\begin{array}{l}k \\
\left(10^{-2} \mathrm{~min}^{-1}\right)\end{array}$ \\
\hline Pc & QD480 & RB & 6.35 \\
Pc & QD530 & RB & 6.65 \\
Pc & None & RB & 1.16 \\
Pa & QD480 & RB & 20.83 \\
Pa & QD530 & RB & 21.52 \\
Pa & None & RB & 1.74 \\
None & QD480 $^{c}$ & None & $<0.01$ \\
None & QD530 $^{c}$ & None & $<0.01$ \\
None & None & RB $^{d}$ & $<0.01$
\end{tabular}

${ }^{a} 4 \mathrm{mg} \mathrm{mL}{ }^{-1}$ in methanol. ${ }^{b} 41.7 \mu \mathrm{g}$ QD per mg polymer. ${ }^{c} 167 \mu \mathrm{g} \mathrm{mL}{ }^{-1}$. ${ }^{d} 5 \mu \mathrm{M}$.

transformation at much higher rates. It is worth noting that there are notable differences between supports $\mathbf{P c}$ and $\mathbf{P a}$. The photocatalysts based on the polymers displaying ethylenediamine pendant groups (Pa) are the most active ones, with rate constants of about $0.2 \mathrm{~min}^{-1}$. Taking into account that polystyrene polymeric matrices are hydrophobic and present an unfavorable swelling in $\mathrm{MeOH}$, these differences can be assigned to a better diffusion of the $\mathrm{MeOH}$ solutions to and from the polymeric surfaces where the photocatalysts are located. Nevertheless, the participation of the aminoalkyl moieties present in Pa in the interaction with the QDs on the surface of the polymer cannot be discarded. On the other hand, it deserves to be mentioned that the size of the QDs has little influence on the photocatalytic performance, since the observed rates are approximately the same irrespective of the nanoparticle used.

Thus, the morphology of the polymeric supports and the interaction between QDs and RB (and/or with other functional groups present in the polymeric matrix) seem to affect to this photocatalytic process. It is not possible, currently to provide a conclusive analysis of the nature of the chemical interaction between both systems, in particular due to the low concentration of both components, which hampers any direct measurement through classical approaches. However, in terms of the photo-physical interaction, the way energy is captured by the QDs and transferred to RB follows the Förster's Resonance Energy Transfer mechanism. ${ }^{8}$

In Table 1 all the data are collected for a better comparison. Additional control irradiations were carried out using only QDs as absorbers (without $\mathbf{R B}$ ) as potential generators of ${ }^{1} \mathrm{O}_{2}$. As it can be seen, QDs alone are not able to produce a detectable amount of ${ }^{1} \mathrm{O}_{2}$, in agreement with literature data. ${ }^{6,7}$

\section{Conclusions}

In summary, it has been demonstrated that, by means of sensitization with core/shell CdSe/ZnS QDs, it is possible to induce the formation of reactive oxygen species, like singlet oxygen, useful for photocatalytic oxygenations, irradiating at a wavelength not favorable for the photosensitizer. This 
phenomenon takes advantage of the broad absorption and high extinction coefficients of these semiconducting nanoparticles combined with the feasibility of an energy transfer to the photosensitizer. This double energy transfer process (QD to RB and $\mathbf{R B}$ to $\mathrm{O}_{2}$ ) could find applications in areas beyond medicine, namely in sustainable chemistry taking into account that this simple procedure extends the range of excitation of most of the described supported organic sensitizers and hence allows a better exploitation of the only unlimited energy source, which is the light of Sun.

\section{Conflicts of interest}

There are no conflicts of interest to declare.

\section{Acknowledgements}

Financial support from Generalitat Valenciana (PROMETEO/ 2016/071) and MINECO (CTQ2015-68429-R) and technical support by SCIC/UJI are acknowledged.

\section{Notes and references}

1 R. E. Galián and J. Pérez-Prieto, Energy Environ. Sci., 2010, 3, 1488; G. Palmisano, V. Augugliaro, M. Pagliaro and L. Palmisano, Chem. Commun., 2007, 3425; A. Albini and M. Fagnoni, Green Chem., 2004, 6, 1; D. Ravelli, M. Fagnoni and A. Albini, Chem. Soc. Rev., 2013, 42, 97; S. Protti and M. Fagnoni, Photochem. Photobiol. Sci., 2009, 8, 1499; A. Albini and M. Fagnoni, ChemSusChem, 2008, 1, 63; M. Oelgemöller, Chem. Rev., 2016, 116, 9664.

2 D. Friedmann, A. Hakki, H. Kim, W. Choi and D. Bahnemann, Green Chem., 2016, 18, 5391; J. M. Herrmann, Appl. Catal., B, 2010, 99, 461; M. Pelaez, N. T. Nolan, S. C. Pillai, M. K. Seery, P. Falaras, A. G. Kontos, P. S. M. Dunlop, J. W. J. Hamilton, J. A. Byrne, K. O'Shea, M. H. Entezari and D. D. Dionysiou, Appl. Catal., B, 2012, 125, 331; D. Spasiano, R. Marotta, S. Malato, P. Fernández-Ibáñez and I. Di Somma, Appl. Catal., B, 2015, 170, 90; S. Malato, J. Blanco, A. Vidal and C. Richter, Appl. Catal., B, 2002, 37, 1; T. Ochiai and A. Fujishima, J. Photochem. Photobiol., C, 2012, 13, 247.

3 S. Lacombe and T. Pigot, Catal. Sci. Technol., 2016, 6, 1571. 4 H. H. Perkampus, UV-VIS Atlas of Organic Compounds, VCH, 1992.

5 D. E. J. G. J. Dolmans, D. Fukumura and R. K. Jain, Nat. Rev. Cancer, 2003, 3, 380; W. Fan, P. Huang and X. Chen, Chem. Soc. Rev., 2016, 45, 6488.

6 A. C. S. Samia, X. Chen and C. Burda, J. Am. Chem. Soc., 2003, 125, 15736.

7 R. Bakalova, H. Ohba, Z. Zhelev, T. Nagase, R. Jose, M. Ishikawa and Y. Baba, Nano Lett., 2004, 4, 1567; L. Shi, B. Hernandez and M. Selke, J. Am. Chem. Soc., 2006, 128, 6278; J. M. Tsay, M. Trzoss, L. Shi, X. Kong, M. Selke, M. E. Jung and S. Weiss, J. Am. Chem. Soc., 2007, 129, 6865; J. Ma, J.-Y. Chen, M. Idowu and T. Nyokong, J. Phys. Chem.
B, 2008, 112, 4465; E. I. Zenkevich, E. I. Sagun, V. N. Knyukshto, A. S. Stasheuski, V. A. Galievsky, A. P. Stupak, T. Blaudeck and T. Borczyskowski, J. Phys. Chem. C, 2011, 115, 21535; G. Charron, T. Stuchinskaya, D. R. Edwards, D. A. Russell and T. Nann, J. Phys. Chem. C, 2012, 116, 9334; J. Suchánek, K. Lang, V. Novakova, P. Zimcik, Z. Zelinger and P. Kubát, Photochem. Photobiol. Sci., 2013, 12, 743; I. V. Martynenko, A. O. Orlova, V. G. Maslov, A. V. Baranov, A. V. Fedorov and M. Artemyev, Beilstein J. Nanotechnol., 2013, 4, 895; J. Valanciunaite, A. S. Klymchenko, A. Skripka, L. Richert, S. Steponkiene, G. Streckyte, Y. Mely and R. Rotomskis, $R S C$ Adv., 2014, 4, 52270; E. Yaghini, F. Giuntini, I. M. Eggleston, K. Suhling, A. M. Seifalian and A. J. MacRobert, Small, 2014, 10, 782; C. M. Lemon, E. Karnas, X. Han, O. T. Burns, T. J. Kempa, D. Fukumura, M. G. Bawendi, R. K. Jain, D. G. Duda and D. G. Nocera, J. Am. Chem. Soc., 2015, 137, 9832; R. Timor, H. Weitman, N. Waiskopf, U. Banin and B. Ehrnberg, ACS Appl. Mater. Interfaces, 2015, 7, 21107; L. Li, J. Liu, X. Yang, Z. Peng, W. Liu, J. Xu, J. Tang, X. He and K. Wang, Chem. Commun., 2015, 51, 14357.

8 E. Yaghini, A. M. Seifalian and A. J. MacRobert, Nanomedicine, 2009, 4, 353; T. Avellini, C. Lincheneau, F. Vera, S. Silvi and A. Credi, Coord. Chem. Rev., 2014, 263264, 151; N. Hildebrandt, C. M. Spillmann, W. R. Algar, T. Pons, M. H. Stewart, E. Oh, K. Susumu, S. A. Díaz, J. B. Delehanty and I. L. Medintz, Chem. Rev., 2017, 117, 536. 9 V. Fabregat, M. I. Burguete, F. Galindo and S. V. Luis, Environ. Sci. Pollut. Res., 2014, 21, 11884.

10 J. Zhao, W. Wu, J. Sun and S. Guo, Chem. Soc. Rev., 2013, 42, 5323; A. G. Griesbeck, T. T. El-Idreesy and A. Bartoschek, Adv. Synth. Catal., 2004, 346, 245; J. Wahlen, D. E. De Vos, P. A. Jacobs and P. L. Alsters, Adv. Synth. Catal., 2004, 346, 152; S. Wang, R. Gao, F. Zhou and M. Selke, J. Mater. Chem., 2004, 14, 487.

11 M. I. Burguete, R. Gavara, F. Galindo and S. V. Luis, Tetrahedron Lett., 2010, 51, 3360; M. I. Burguete, R. Gavara, F. Galindo and S. V. Luis, Catal. Commun., 2010, 11, 1081; A. A. Ghogare and A. Greer, Chem. Rev., 2016, 116, 9994; M. C. DeRosa and R. J. Crutchley, Coord. Chem. Rev., 2002, 233, 351; A. P. Schaap, A. L. Thayer, E. C. Bloosey and D. C. Neckers, J. Am. Chem. Soc., 1975, 97, 3741.

12 A. Beltrán, M. Mikhailov, M. N. Sokolov, V. Pérez-Laguna, A. Rezusta, M. J. Revillo and F. Galindo, J. Mater. Chem. B, 2016, 4, 5975; M. I. Burguete, F. Galindo, R. Gavara, S. V. Luis, M. Moreno, P. Thomas and D. A. Russell, Photochem. Photobiol. Sci., 2009, 8, 37; P. R. Ogilby, Chem. Soc. Rev., 2010, 39, 3181; Singlet Oxygen: Applications in Biosciences and Nanosciences, ed. S. Nonell and C. Flors, Royal Society of Chemistry, 2016.

13 M. L. Marín, L. Santos-Juanes, A. Arques, A. M. Amat and M. A. Miranda, Chem. Rev., 2012, 112, 1710; A. Yavorskyy, O. Shvydkiv, C. Limburg, K. Nolan, Y. M. C. Delauré and M. Oelgemöller, Green Chem., 2012, 14, 888.

14 A. Beltrán, M. I. Burguete, S. V. Luis and F. Galindo, RSC $A d v .$, 2016, 6, 56064; P. D. Wadhavane, R. E. Galián, 
M. A. Izquierdo, J. Aguilera-Sigalat, F. Galindo, L. Schmidt, M. I. Burguete, J. Pérez-Prieto and S. V. Luis, J. Am. Chem. Soc., 2012, 134, 20554; P. D. Wadhavane, M. A. Izquierdo, M. I. Burguete, F. Galindo and S. V. Luis, Soft Matter, 2012,
8, 4373; V. Fabregat, M. A. Izquierdo, M. I. Burguete, F. Galindo and S. V. Luis, Inorg. Chim. Acta, 2012, 381, 212; J. Rubio, M. A. Izquierdo, M. I. Burguete, F. Galindo and S. V. Luis, Nanoscale, 2011, 3, 3613. 